\title{
Mechanically Reconfigurable Microstrip Lines Loaded with Stepped Impedance Resonators and Potential Applications
}

\author{
J. Naqui and F. Martín \\ GEMMA/CIMITEC, Departament d'Enginyeria Electrònica, Universitat Autònoma de Barcelona, 08193 Bellaterra, Spain \\ Correspondence should be addressed to F. Martín; ferran.martin@uab.es
}

Received 15 January 2014; Accepted 16 January 2014; Published 20 February 2014

Academic Editor: Giacomo Oliveri

Copyright (C) 2014 J. Naqui and F. Martín. This is an open access article distributed under the Creative Commons Attribution License, which permits unrestricted use, distribution, and reproduction in any medium, provided the original work is properly cited.

\begin{abstract}
This paper is focused on exploring the possibilities and potential applications of microstrip transmission lines loaded with stepped impedance resonators (SIRs) etched on top of the signal strip, in a separated substrate. It is shown that if the symmetry plane of the line (a magnetic wall) is perfectly aligned with the electric wall of the SIR at the fundamental resonance, the line is transparent. However, if symmetry is somehow ruptured, a notch in the transmission coefficient appears. The notch frequency and depth can thus be mechanically controlled, and this property can be of interest for the implementation of sensors and barcodes, as it is discussed.
\end{abstract}

\section{Introduction}

The topic of metamaterials has experienced an exponential growth in the field of science and technology since 2000, when the first metamaterial structure (a one-dimensional left-handed medium) was reported [1]. In particular, metamaterial transmission lines (first reported in [2-4]) have attracted the attention of RF/microwave engineers, since such artificial lines exhibit further controllability than ordinary lines, and they can be used as building blocks for the implementation of microwave devices with small size and/or high performance and with novel functionalities as well [57].

There are nowadays many different approaches and types of metamaterial transmission lines. Among them, the socalled resonant type approach has been revealed to be very useful for the implementation of microwave components (including filters, dividers, and leaky wave antennas) [7]. Resonant type metamaterial transmission lines are implemented by loading a host line with electrically small resonant elements, typically, although not exclusively, split ring resonators (SRRs) $[8,9]$ and complementary split ring resonators (CSRRs) [10]. By loading a host line with a coupled (electrically, magnetically, or both) resonator, a notch in the transmission coefficient (at the fundamental frequency) appears, and such notch widens if the line is loaded with several resonators. The stopband characteristic exhibited in a transmission line loaded with an array of electrically small resonators has been interpreted as due to the negative effective permeability (SRRs) or permittivity (CSRRs) of the lines [9-11].

However, it is not necessary to invoke metamaterials theory to interpret and understand the stopband functionality of SRR or CSRR loaded lines. In most applications, the lines are loaded with a single resonator stage because this reduces size or suffices to achieve the required functionality. Indeed the modeling through equivalent circuits of transmission lines loaded with electrically small resonators, such as those aforementioned, has been a subject of an intensive study in the last years [11-16]. Certainly, transmission lines loaded with a single or with few resonators cannot be considered to be effective media metamaterials. However, in certain applications, the controllability of the dispersion and characteristic impedance of the loaded lines are fundamental to achieve the required functionality, and for this reason such lines are typically referred to as metamaterial transmission lines [17]. In other applications, the key point is to achieve stopband functionality. In these cases, as long as the considered resonators are electrically small and potentially useful 


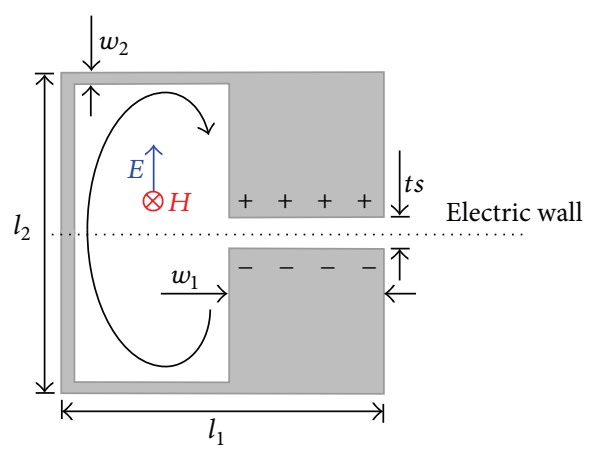

FIGURE 1: Typical topology of a folded SIR and relevant dimensions. The resonator can be excited by a time varying electric or magnetic field oriented in the indicated directions. The distribution of current and charges is also sketched.

for the implementation of effective media metamaterials, the lines are usually designated as transmission lines with metamaterial loading. The structures considered in this work belong to this category: microstrip lines loaded with stepped impedance resonators (SIRs). We have considered both folded SIRs and conventional SIRs coupled to the lines and separated from the signal strip of the line by means of a dielectric slab. It will be seen that the response of the SIR loaded lines can be tailored through mechanical actuation and potential applications of this effect are discussed.

\section{Microstrip Lines Loaded with Stepped Impedance Resonators (SIRs)}

Stepped impedance resonators are common planar resonators in microwave engineering [18]. Essentially SIRs are metallic open-ended strip resonators where the width of the strip is varied abruptly. In a trisection SIR, it is well known that by narrowing the central section and widening the external ones, the resonator can be made electrically small as compared to a uniform half wavelength resonator [18]. Additional miniaturization can be achieved by folding the SIR, as Figure 1 illustrates. It is important to mention, for the purposes of this paper, that the SIR exhibits an electric wall at the indicated (symmetry) plane (see Figure 1) at the fundamental resonance. Hence, the resonator exhibits an electric dipole perpendicular to the symmetry plane, and it can be excited through an electric field oriented in that direction. Moreover, in a folded SIR, as the one depicted in Figure 1, the current loop induces a magnetic dipole moment and therefore the resonator can also be magnetically driven (this is not the case in unfolded SIRs).

According to the previous statements, in a transmission line loaded with a folded SIR, the line-to-resonator coupling may be electric, magnetic, or mixed, whereas in a transmission line loaded with an unfolded SIR the magnetic coupling is prevented. For instance, it was demonstrated in [19] that in coplanar waveguide (CPW) transmission lines loaded with folded SIRs on the back side of the substrate (with meaningful line-to-resonator capacitances and thin substrates), the dominant line-to-resonator coupling mechanism is electric.

Regardless of the coupling mechanism, if both the resonator and the line are symmetric, there is a necessary condition for particle excitation: either the symmetry planes of the line and resonator are not aligned, or, if they are aligned, they must be of the same electromagnetic nature (either an electric wall or a magnetic wall). In other words, if the symmetry planes of the line and resonator are aligned and they are of distinct electromagnetic nature, then electromagnetic coupling capable of exciting the resonator does not arise. The result is a transmission coefficient for the loaded line close to 1 even at the fundamental resonance of the considered particle. This operating principle is universal, being independent of the line type, the resonator, and the resonator-to-line coupling mechanism $[19,20]$.

To gain more insight into this feature, let us consider a symmetric structure consisting of a microstrip line loaded with a folded SIR on top of the signal strip, with aligned symmetry planes. As mentioned above, the particle cannot be excited at the fundamental resonance, by neither the magnetic field nor the electric field generated by the line. Due to symmetry, the magnetic field components at both sides of the symmetry plane of the particle are perfectly cancelled, and the magnetic field is not able to induce circulating currents in the SIR. Analogously, for electric field excitation, a net component of the electric field orthogonal to the symmetry plane is required, and this is impossible due to the symmetry of the line and to the fact that the symmetry plane of the line is a magnetic wall. However, if the symmetry is broken, the perfect cancellation of fields vanishes, the particle is excited, and the incident power is partially reflected at the fundamental resonance, thus providing a notch in the transmission coefficient at that frequency.

\section{Mechanical Reconfigurability of the SIR Loaded Microstrip Lines and Possible Applications}

In order to symmetrically load a microstrip line with an SIR, three metal levels are required. A possible multilayer structure is the one depicted in Figure 2, with top and bottom substrates. To mechanically rupture the symmetry of the structure and thus achieve a notch in the transmission coefficient, there are several possibilities. For instance, the SIR and the line can be etched on different (movable) substrates with a relative motion. Thus, by laterally displacing the top substrate, symmetry is broken. Another interesting approach to rupture symmetry is by implementing the SIRs with deflectable cantilever type (movable) arms through microelectromechanical systems (MEMS) (similar to the reconfigurable SRR loaded lines reported in [21]). Any physical variable able to deflect the suspended parts of the SIR can potentially be detected/sensed through its effect on the transmission coefficient of the loaded line. Of course the MEMS-based SIRs can be actuated electronically, with the potential to implement reconfigurable notch filters. However, it is also possible to break symmetry through the effects of an external 


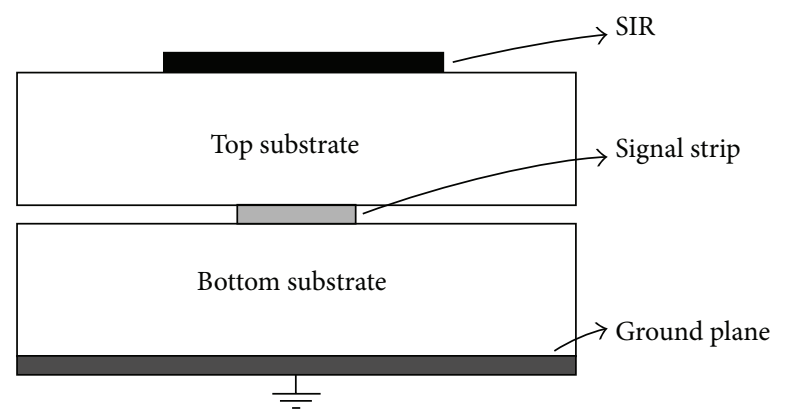

FIgURE 2: Cross-sectional view of a microstrip line loaded with an SIR, etched on top of it.

force, pressure, torque, and so forth. Alternatively, the effects of these variables can also be potentially sensed/detected by using malleable/flexible substrates, rather than deflectable SIRs. This eases the fabrication process and reduces costs. Finally, dielectric loading may be another strategy to rupture symmetry. The idea behind this approach is to add a material or substance on top of the SIR or in specific test regions of the structure (for instance, by creating symmetric holes inside the top substrate). As long as the material or substance exhibits a symmetric distribution of the dielectric permittivity, the line is transparent to signal propagation. However, if the dielectric loading is not symmetric, this can be detected by the appearance of a notch in the transmission coefficient. One possibility that this approach opens concerns the detection of defects or imperfections in certain substances by comparison to a reference or standard. This approach may be useful for analysis of substances, including organic tissues and microfluidics. Sensors for analysis of organic tissues and dielectric monitoring of microfluidic channels based on the frequency or quality-factor variation of resonant elements have recently been proposed (see, e.g., [22-24]).

\section{Illustrative Preliminary Examples}

In this section, two application examples of SIR loaded microstrip lines are reported. Firstly, the symmetry properties of such loaded lines are exploited as an encoding mechanism to implement an RF (radiofrequency) barcode. Secondly, the symmetry rupture is used as a sensing mechanism to design an alignment/displacement sensor.

4.1. RF Barcode. A multiresonator-based chipless radiofrequency identification (RFID) tag is an RF barcode where the information is stored in the so-called spectral signature of the tag. Such spectral signature is obtained by loading a transmission line with several resonators, each resonating at a different frequency [25]. Each resonator corresponds to a bit, and the logic " 1 " or " 0 " is chosen by the presence or absence of the notch at the resonance frequency (i.e., the encoding is in amplitude). By coupling the resonator to the line, the notch appears, giving the logic level "1." On the contrary, the notch can be prevented (logic level " 0 ") by removing or short-circuiting the resonator (this shifts the resonance frequency outside the region of interest) [25]. An alternative to these conventional approaches is to align the symmetry planes of the line and resonator. Thus, by using a microstrip line loaded with folded SIRs, if the resonator symmetry plane is aligned with that of the line, the resonator is not coupled to the line and the notch at the fundamental resonance does not arise. Conversely, the logic "1" can be achieved, for instance, by laterally displacing the resonator. Under these conditions, the magnetic wall of the line and the electric wall of the resonator are not aligned, and the resonator is excited. Interestingly, as long as the SIRs can be laterally shifted independently, a reconfigurable barcode is potentially possible. Figure 3(a) depicts the top view of a microstrip line loaded with three folded SIRs, each designed to resonate at a different frequency. Notice that in this configuration the central SIR is aligned with the line, whereas the SIRs of the extremes are nonaligned. Therefore the line is encoded with the code "101." Figure 3(b) depicts the simulated frequency response (i.e., the spectral signature) of this structure and also the one corresponding to the complementary code, that is, "010," where the aligned SIRs are those of the extremes.

In the proposed barcode, the ground plane is kept unaltered, this being an advantage as compared to other proposals [26]. An important aspect to highlight is that the design of the line and SIRs does not require special attention, since the main purpose is to achieve narrow notches. This allows accommodating the maximum number of resonators (resonance frequencies) in a predefined frequency spectrum, thus embedding as many bits as possible. Narrow notches typically arise if the coupling between the line and the resonator is weak, as shown in the structure of Figure 3 (the reason is that the dominant electric coupling is poor because of the extremely narrow line). Conversely, in the next example, the aim is to obtain a high sensitivity of the notch magnitude (depth) with the lateral displacement, and this requires a specific design.

4.2. Alignment/Position Sensor. Let us now consider the application of SIR loaded microstrip lines to detect lack of lateral alignment or to measure lateral shift between two surfaces with relative motion to each other (other proposals can be found in $[27,28]$ ). In this case, a figure of merit is the sensitivity of the notch magnitude and/or frequency with the lateral displacement. For the implementation of these sensors, it is critical to adequately choose the topology of 


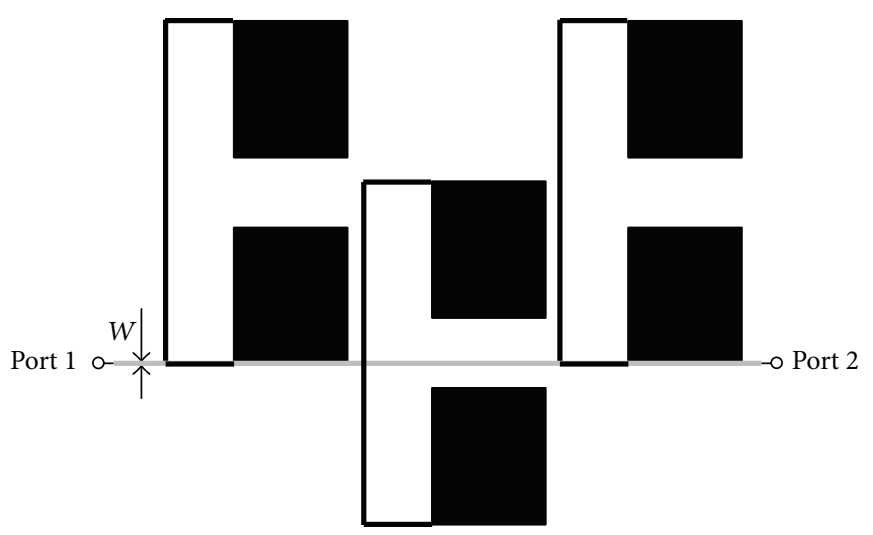

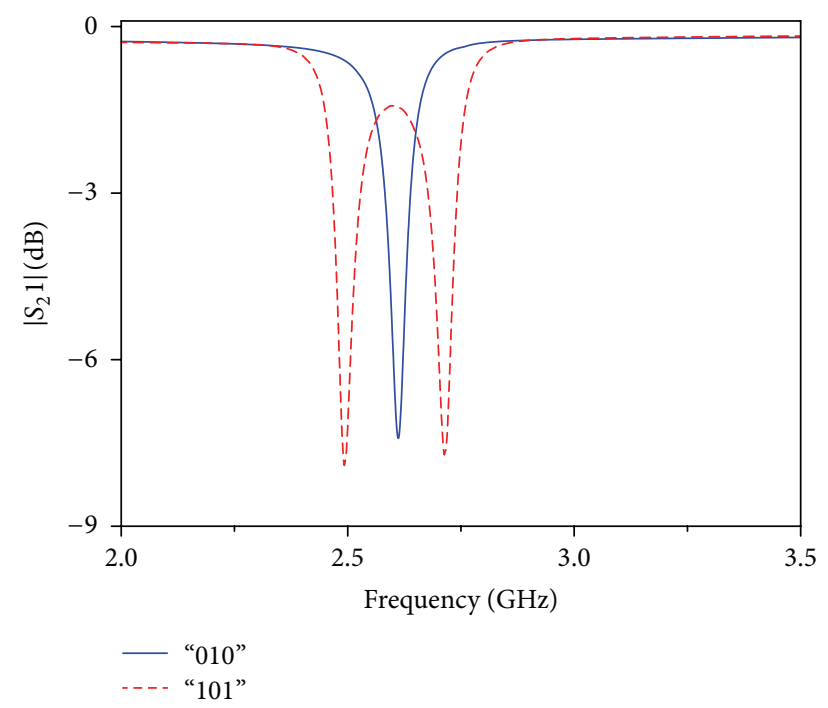

(b)

Figure 3: Microstrip line loaded with folded SIRs for the code "101" (a) and lossy electromagnetic simulation of the transmission coefficient corresponding to the indicated codes (b). The top and bottom substrates are Rogers RO3010 with thickness $h=0.254 \mathrm{~mm}$, dielectric constant $\varepsilon_{r}=11.2$, and loss tangent $\tan \delta=0.0023$. The dimensions are line width $W=0.2 \mathrm{~mm}$, corresponding to a characteristic impedance close to $50 \Omega$; for the folded SIRs, $w_{1}(2.5 \mathrm{GHz})=2 \mathrm{~mm}, w_{1}(2.6 \mathrm{GHz})=1.8 \mathrm{~mm}, w_{1}(2.7 \mathrm{GHz})=1.64 \mathrm{~mm}, w_{2}=0.2 \mathrm{~mm}, s=1.2 \mathrm{~mm}, l_{1}(2.5 \mathrm{GHz})=$ $3.26 \mathrm{~mm}, l_{1}(2.6 \mathrm{GHz})=3.06 \mathrm{~mm}, l_{1}(2.7 \mathrm{GHz})=2.9 \mathrm{~mm}$, and $l_{2}=6 \mathrm{~mm}$.

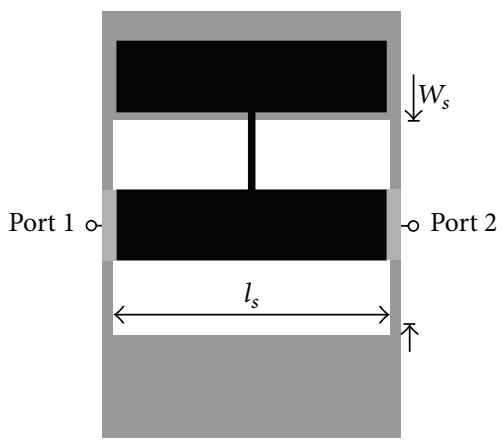

(a)

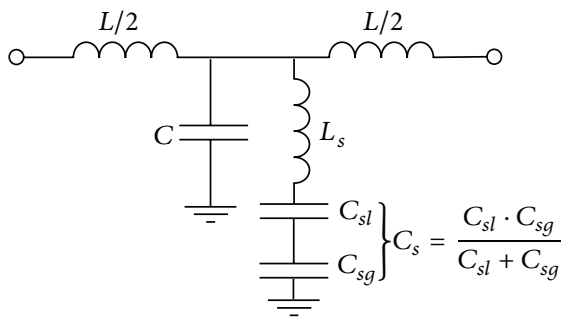

(b)

FIgURE 4: Proof of concept demonstrator of an SIR loaded microstrip line useful as alignment/position sensor for a displacement corresponding to the strongest notch (a) and lumped element equivalent circuit model (b). The microstrip line dimensions are $W=2 \mathrm{~mm}$, $W_{s}=5.8 \mathrm{~mm}$, and $l_{s}=7.8 \mathrm{~mm}$; the lengths of the SIR sections are $2 \mathrm{~mm}$, and the widths are $w_{1}=7.6 \mathrm{~mm}$ and $w_{2}=0.2 \mathrm{~mm}$. The top and bottom substrates are Rogers RO3010 with $h=0.254 \mathrm{~mm}, \varepsilon_{r}=11.2$, and $\tan \delta=0.0023$. The inductance and capacitance of the line section are $L$ and $C$, the inductance of the SIR is $L_{s}$, the SIR-to-line capacitance is $C_{s l}$, and the SIR-to-ground capacitance is $C_{s g}$.

both the SIR and the line. In order to boost the electric coupling between the line and the SIR, it is important to enhance the patch capacitance of the SIR to the line (as well as to the ground), and for this reason the line width must be widened. Since this reduces the line characteristic impedance, in order to match the ports to $50 \Omega$, a window in the ground plane below the position of the signal strip can be etched. This enhances the line inductance and drops the line capacitance simultaneously, compensating the reduction of the characteristic impedance. Moreover, since the electric coupling is generally stronger than magnetic coupling in SIRs, we have opted to use an unfolded configuration. Specifically, we have designed the proof of concept prototype shown in Figure 4(a), corresponding to the lateral shift providing the widest and deepest notch. To enhance the electric coupling for nonsymmetric loadings, the width of the line has been chosen to be identical to the length of the wide strip sections of the SIR. We have thus etched a slot in the ground plane (see the dimensions in Figure 4) to achieve good matching to $50 \Omega$ in the symmetrically loaded line.

A lumped element equivalent circuit model of the structure of Figure 4(a) is proposed and depicted in Figure 4(b). According to this simple model, it is clear that to strengthen the notch it is necessary to increase the ratio between the resulting patch capacitance, $C_{s}$ (given by the capacitance between the SIR and line, $C_{s l}$, and by that between the 


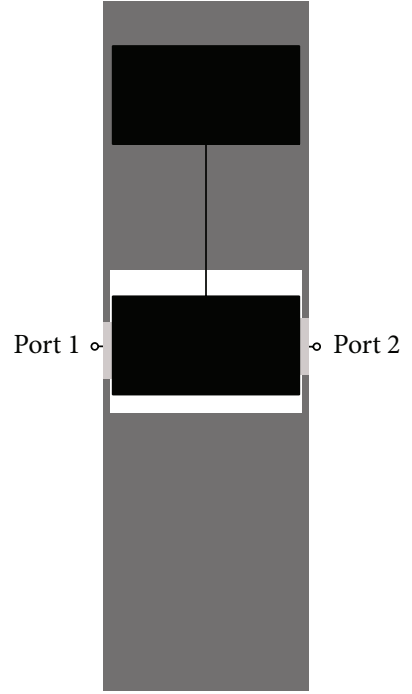

(a)

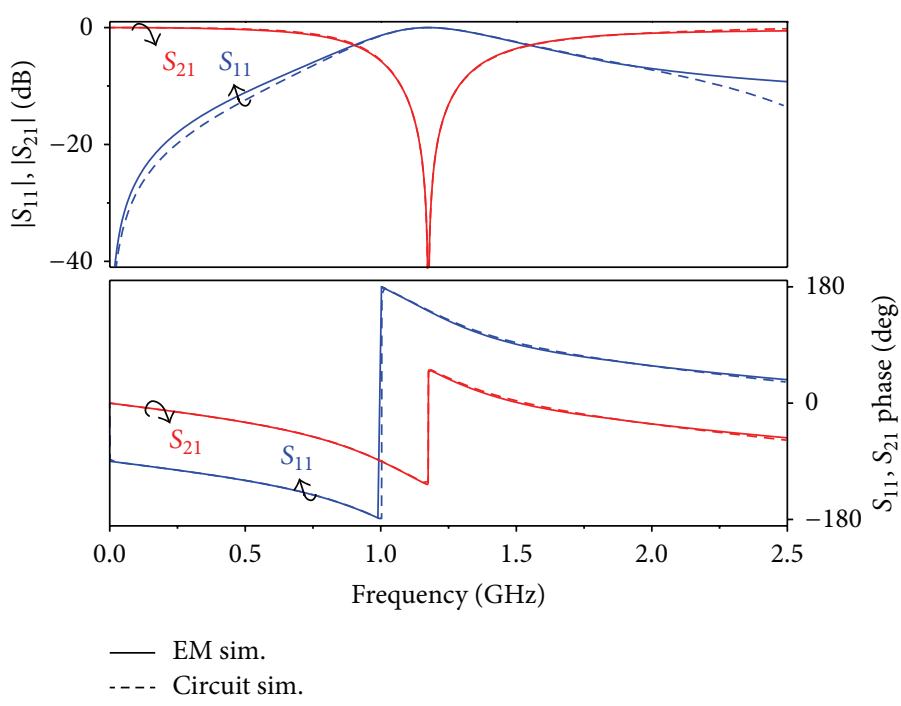

(b)

FIGURE 5: Topology of an electrically small SIR loaded microstrip line (a) and transmission/reflection coefficients from the lossless electromagnetic and circuit simulations (b). The structure is the same as that of Figure 4(a) with the exception of the SIR length, with the lengths of the wide and narrow sections being, respectively, $4 \mathrm{~mm}$ and $6 \mathrm{~mm}$. The lumped element values are $L=4.43 \mathrm{nH}, C=1.58 \mathrm{pF}$, $L_{s}=6.90$, and $C_{s}=2.66 \mathrm{pF}$.

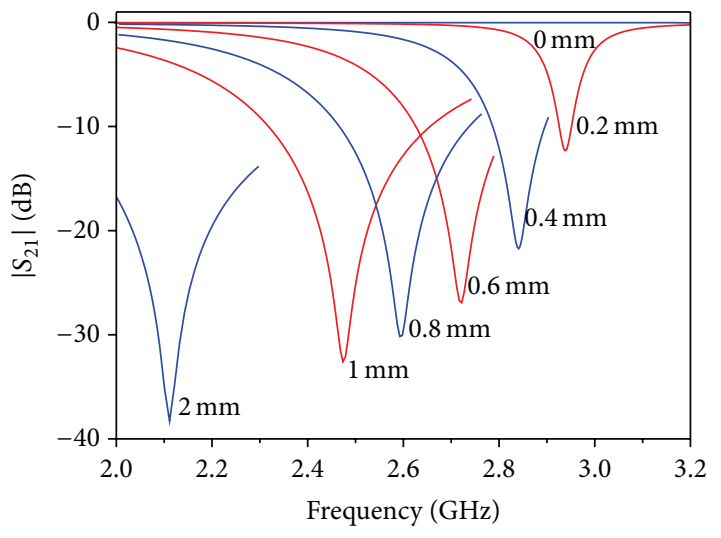

(a)

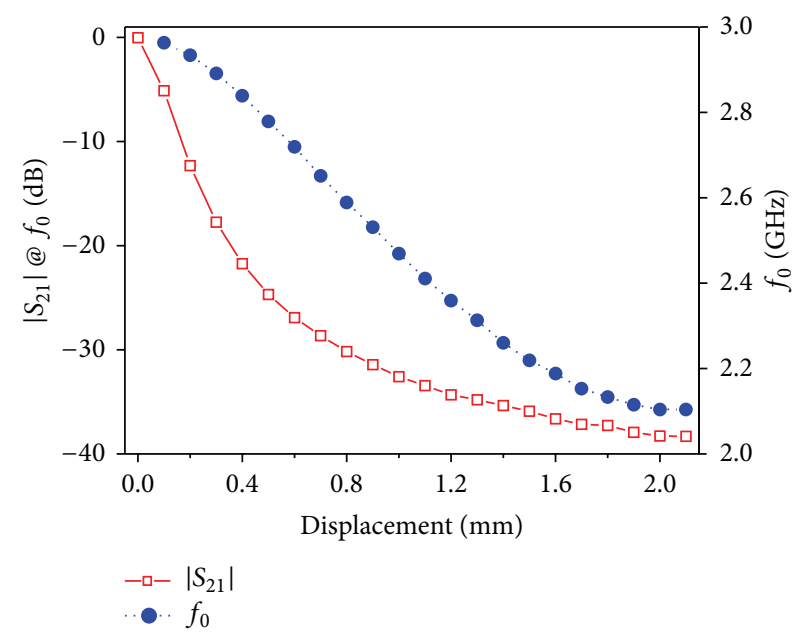

(b)

FiguRE 6: Transmission coefficient (a) and dependence of the notch magnitude and frequency (b) with lateral displacement corresponding to the structure of Figure 4, inferred from the lossy electromagnetic simulation. The displacement for maximum notch magnitude, that is, the dynamic range, is $2 \mathrm{~mm}$.

SIR and ground, $C_{s g}$ ), and the SIR inductance, $L_{s}$. Hence, the equivalent circuit confirms that the electric coupling should be enhanced as much as possible, and for this reason the step in width in the considered SIR is substantially extreme. With a view to validate the circuit model, we have increased the inductance $L_{s}$ and the capacitance $C_{s}$ by lengthening, respectively, the narrow and the wide SIR sections, as shown in Figure 5(a). By this means the unit cell length is shortened at the resonance frequency and the circuit model is expected to predict the frequency response of the structure more accurately (note that this is at the expense of sensitivity due to the increase in the transversal dimension). The circuit parameters have been extracted in an analogous manner as in the procedure reported in [29]. In particular, such systematic method is based on a mapping from the electromagnetic simulation to the circuit response as follows: (i) the impedance of the series resonator $L_{s}-C_{s}$ nulls at the notch frequency, (ii) when the series and shunt impedances are conjugate the transmission coefficient phase is $-90^{\circ}$, and (iii) the intersection between the reflection coefficient and the unit normalized resistance circle provides the resonance frequency of the parallel resonator $C-L_{s}-C_{s}$ and also (iv) the 

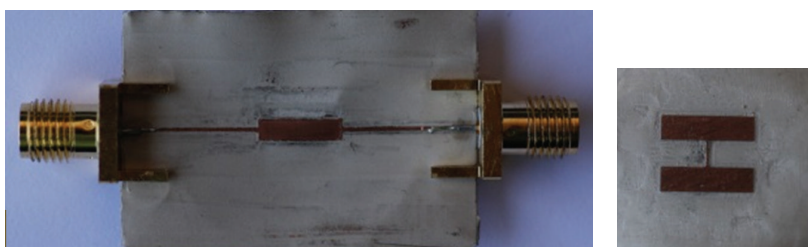

(a)
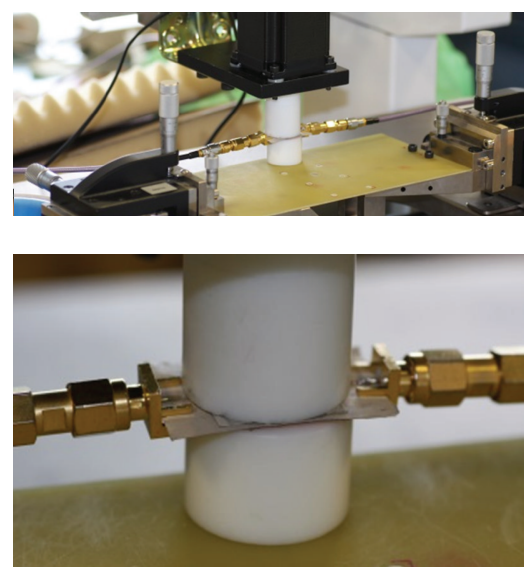

(b)

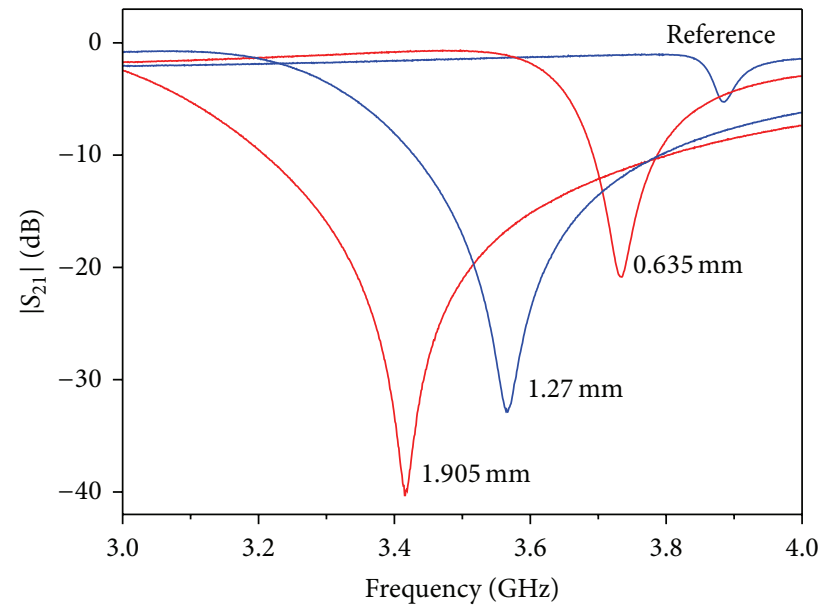

(c)

FIGURE 7: Photograph of the fabricated SIR loaded microstrip line of Figure 4 on separate substrates, where matched input/output lines of $W=0.2 \mathrm{~mm}$ have been added to solder the connectors (a). Photograph of the experimental setup with the substrates attached to Teflon slabs (a material with a low dielectric constant, $\varepsilon_{r}=$ 2.08) and with manual positioners labeled in inches (b). Measured transmission coefficient for some lateral displacements of the SIR (c).

series inductance $L$. As can be appreciated in Figure 5(b), the circuit simulation fits the lossless electromagnetic simulation in a very good approximation.

Figure 6(a) plots the transmission coefficient inferred from the lossy electromagnetic simulation of the proof of concept structure shown in Figure 6(a) for different lateral displacements. The notch magnitude and frequency versus the displacement were recorded and are indicated in Figure 6(b). As can be observed, both electrical variables are quite sensitive to the displacement, indicative of a significant increase in the capacitive line-to-SIR-to-ground coupling with the displacement. Note that the sensitivity is particularly very high in magnitude for small displacements, and hence the sensor is very suitable for alignment purposes. Specifically, the average sensitivity in frequency is $42.95 \mathrm{MHz} / 100 \mu \mathrm{m}$ in the full dynamic range, while in magnitude is $-5.42 \mathrm{~dB} / 100 \mu \mathrm{m}$ in the range up to $400 \mu \mathrm{m}$.

The previous proof of concept demonstrator was fabricated and measured in an experimental setup that enables 3D calibrations through manual positioners (see Figure 7). Thus, the measurement of the transmission coefficient was carried out for different displacements, manually performed. Note that it was not possible to perfectly align the SIR with the line due to the fact that the sensor features a very high sensitivity for small shifts (for this reason a measured sample close to the symmetric structure was labeled as the reference one). Furthermore, the measured notch frequencies were relatively much higher than those obtained by electromagnetic simulations, and this is primarily attributed to a drop of the capacitance $C_{s}$ caused by a thin air layer between the top and bottom substrates (indeed further simulations have suggested that an in-between air layer of $75 \mu \mathrm{m}$ shifts the notch frequency upwards up to the measured results). Nevertheless, the proof of concept is experimentally validated. In the near future, we plan to characterize the structure by considering a specific distance between the line and the SIR (using a setup similar to that reported in [30]). It is also worth to bear in mind the acquisition of a more sophisticated experimental setup to perform $3 \mathrm{D}$ calibrations with higher accuracy.

To end this section, we would like to mention that the notch magnitude and spectral position are also sensitive to the force applied on top of the SIR substrate. This opens the possibility to implement force or pressure sensors based on the reported approach.

\section{Conclusions}

In conclusion, it has been shown that the SIRs are useful resonant particles as loading elements in microstrip lines aimed to act as microwave sensors or RF barcodes based on symmetry rupture. Specifically, for sensing purposes, SIRs provide high sensitivity due to the strong electric coupling between the line and the resonator. This is an advantage over other proposed sensors based on symmetry properties (e.g., displacement sensors that use split ring resonators). Moreover, these SIR loaded lines can also be potentially useful for other types of sensors, such as pressure sensors, or sensors for dielectric characterization, where the high sensitivity of SIR loaded lines can represent an advantage over other exiting approaches. Work is in progress in this regard. Concerning barcodes, a proof of concept has been developed. Although the number of bits achievable with the reported approach is limited (unless the spectral bandwidth is very high), tags based on the reported approach can be of interest in systems that do not require to store too much information and do not need a high level of security. Moreover, these 
tags can be programmable (symmetry can be achieved by adding metallic strips to the SIRs) and can be implemented in flexible substrates by means of printed techniques (including low cost paper substrates). Thus, the potential can be very high (they can be useful, e.g., for document identification, product identification, electoral processes with small voting members, etc.). In summary, several applications of SIR loaded lines have been discussed, and a proof of concept (experimentally validated) of an alignment/position sensor has been provided.

\section{Conflict of Interests}

The authors declare that there is no conflict of interests regarding the publication of this paper.

\section{Acknowledgments}

This work has been supported by MINECO (Spain) (Projects TEC2010-17512, CSD2008-00066, and TEC2011-13615-E) and by AGAUR (Generalitat de Catalunya), through Project 2009SGR-421. Jordi Naqui is also in debt to MECD (Spain) for supporting his work through the FPU Grant AP2010-0431.

\section{References}

[1] D. R. Smith, W. J. Padilla, D. C. Vier, S. C. Nemat-Nasser, and S. Schultz, "Composite medium with simultaneously negative permeability and permittivity," Physical Review Letters, vol. 84, no. 18 , pp. 4184-4187, 2000.

[2] A. K. Iyer and G. V. Eleftheriades, "Negative refractive index metamaterials supporting 2-D waves," in Proceedings of the IEEE MSS-S International Microwave Symposium Digest, pp. 412-415, Seattle, Wash, USA, June 2002.

[3] A. A. Oliner, "A periodic-structure negative-refractive-index medium without resonant elements," in Proceedings of the IEEE AP-S International Symposium and USNC/URSI National Radio Science Meeting, San Antonio, Tex, USA, June 2002.

[4] C. Caloz and T. Itoh, "Application of the transmission line theory of left-handed ( $\mathrm{LH})$ materials to the realization of a microstrip 'LH line," in Proceedings of the IEEE Antennas and Propagation Society International Symposium, vol. 2, pp. 412415, San Antonio, Tex, USA, June 2002.

[5] G. V. Eleftheriades and K. G. Balmain, Negative Refraction Metamaterials: Fundamental Principles and Applications, John Wiley and Sons, New Jersey, NJ, USA, 2005.

[6] C. Caloz and T. Itoh, Electromagnetic Metamaterials: Transmission Line Theory and Microwave Applications, John Wiley and Sons, 2006.

[7] R. Marqués, F. Martín, and M. Sorolla, Metamaterials with Negative Parameters: Theory, Design and Microwave Applications, John Wiley and Sons, 2008.

[8] J. B. Pendry, A. J. Holden, D. J. Robbins, and W. J. Stewart, "Magnetism from conductors and enhanced nonlinear phenomena," IEEE Transactions on Microwave Theory and Techniques, vol. 47, no. 11, pp. 2075-2084, 1999.

[9] F. Martín, J. Bonache, F. Falcone, M. Sorolla, and R. Marqués, "Split ring resonator-based left-handed coplanar waveguide," Applied Physics Letters, vol. 83, no. 22, pp. 4652-4654, 2003.
[10] F. Falcone, T. Lopetegi, J. D. Baena, R. Marqués, F. Martín, and M. Sorolla, "Effective negative- $\varepsilon$ stopband microstrip lines based on complementary split ring resonators," IEEE Microwave and Wireless Components Letters, vol. 14, no. 6, pp. 280-282, 2004.

[11] J. D. Baena, J. Bonache, F. Martín et al., "Equivalent-circuit models for split-ring resonators and complementary splitring resonators coupled to planar transmission lines," IEEE Transactions on Microwave Theory and Techniques, vol. 53, no. 4, pp. 1451-1460, 2005.

[12] L. J. Roglá, J. Carbonell, and V. E. Boria, "Study of equivalent circuits for open-ring and split-ring resonators in coplanar waveguide technology," IET Microwaves, Antennas and Propagation, vol. 1, no. 1, pp. 170-176, 2007.

[13] F. Aznar, J. Bonache, and F. Martín, "Improved circuit model for left-handed lines loaded with split ring resonators," Applied Physics Letters, vol. 92, no. 4, Article ID 043512, 2008.

[14] J. Bonache, M. Gil, O. García-Abad, and F. Martín, "Parametric analysis of microstrip lines loaded with complementary split ring resonators," Microwave and Optical Technology Letters, vol. 50, no. 8, pp. 2093-2096, 2008.

[15] J. Naqui, M. Durán-Sindreu, A. Fernández-Prieto, F. Mesa, F. Medina, and F. Martín, "Multimode propagation and complex waves in CSRR-based transmission line metamaterials," IEEE Antennas and Wireless Propagation Letters, vol.11, pp. 1024-1027, 2012.

[16] J. Naqui, M. Durán-Sindreu, and F. Martín, "Modeling split ring resonator (SRR) and complementary split ring resonator (CSRR) loaded transmission lines exhibiting cross polarization effects," IEEE Antennas and Wireless Propagation Letters, vol. 12, pp. 178-181, 2013.

[17] M. Durán-Sindreu, A. Vélez, G. Sisó et al., "Recent advances in metamaterial transmission lines based on split rings," Proceedings of the IEEE, vol. 99, pp. 1701-1710, 2011.

[18] M. Makimoto and S. Yamashita, "Compact bandpass filters using stepped impedance resonators," Proceedings of the IEEE, vol. 67, no. 1, pp. 16-19, 1977.

[19] J. Naqui, M. Durán-Sindreu, and F. Martín, “On the symmetry properties of coplanar waveguides loaded with symmetric resonators: analysis and potential applications," in Proceedings of the IEEE MTT-S International Microwave Symposium digest, Montreal, Canada, June 2012.

[20] J. Naqui, A. Fernández-Prieto, M. Durán-Sindreu et al., “Common mode suppression in microstrip differential lines by means of complementary split ring resonators: theory and applications," IEEE Transactions on Microwave Theory and Techniques, vol. 60, pp. 3023-3034, 2012.

[21] D. Bouyge, D. Mardivirin, J. Bonache et al., "Split ring resonators (SRRs) based on micro-electro-mechanical deflectable cantilever-type rings: application to tunable stopband filters," IEEE Microwave and Wireless Components Letters, vol. 21, no. 5, pp. 243-245, 2011.

[22] M. Puentes, C. Weiss, M. Schüssler, and R. Jakoby, "Sensor array based on split ring resonators for analysis of organic tissues," in Proceedings of the IEEE MTT-S International Microwave Symposium Digest, Baltimore, Md, USA, June 2011.

[23] M. Schüßeler, C. Mandel, M. Puentes, and R. Jakoby, "Metamaterial inspired microwave sensors," IEEE Microwave Magazine, vol. 13, no. 2, pp. 57-68, 2012. 
[24] T. Chretiennot, D. Dubuc, and K. Grenier, "Optimized electromagnetic interaction microwave resonator/microfluidic channel for enhanced liquid bio-sensor," in Proceedings of the European Microwave Conference, Nuremberg, Germany, October 2013.

[25] S. Preradovic and N. C. Karmakar, "Chipless RFID: bar code of the future," IEEE Microwave Magazine, vol. 11, no. 7, pp. 87-97, 2010.

[26] J. Naqui, M. Durán-Sindreu, and F. Martín, "Differential and single-ended microstrip lines loaded with slotted magnetic-LC resonators," International Journal of Antennas and Propagation, vol. 2013, Article ID 640514, 8 pages, 2013.

[27] C. Mandel, B. Kubina, M. Schüssler, and R. Jakoby, "Passive chipless wireless sensor for two-dimensional displacement measurement," in Proceedings of the 41st European Microwave Conference, pp. 79-82, Manchester, UK, October 2011.

[28] A. Horestani, C. Fumeaux, S. Al-Sarawi, and D. Abbott, "Displacement sensor based on diamond-shaped tapered split ring resonator," IEEE Sensors Journal, vol. 13, no. 4, pp. 1153-1160, 2013.

[29] J. Bonache, M. Gil, I. Gil, J. Garcia-García, and F. Martín, “On the electrical characteristics of complementary metamaterial resonators," IEEE Microwave and Wireless Components Letters, vol. 16, pp. 543-545, 2006.

[30] J. Naqui and F. Martín, “Transmission lines loaded with bisymmetric resonators and their application to angular displacement and velocity sensors," IEEE Transactions on Microwave Theory and Techniques, vol. 61, no. 12, pp. 4700-4713, 2013. 

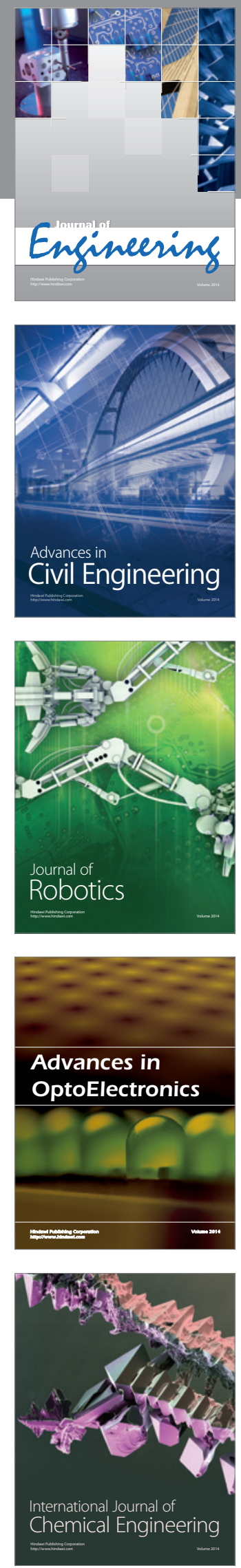

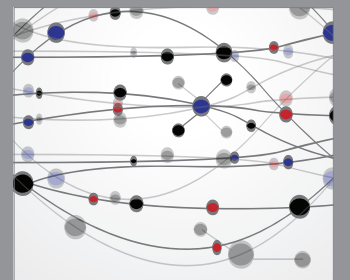

The Scientific World Journal
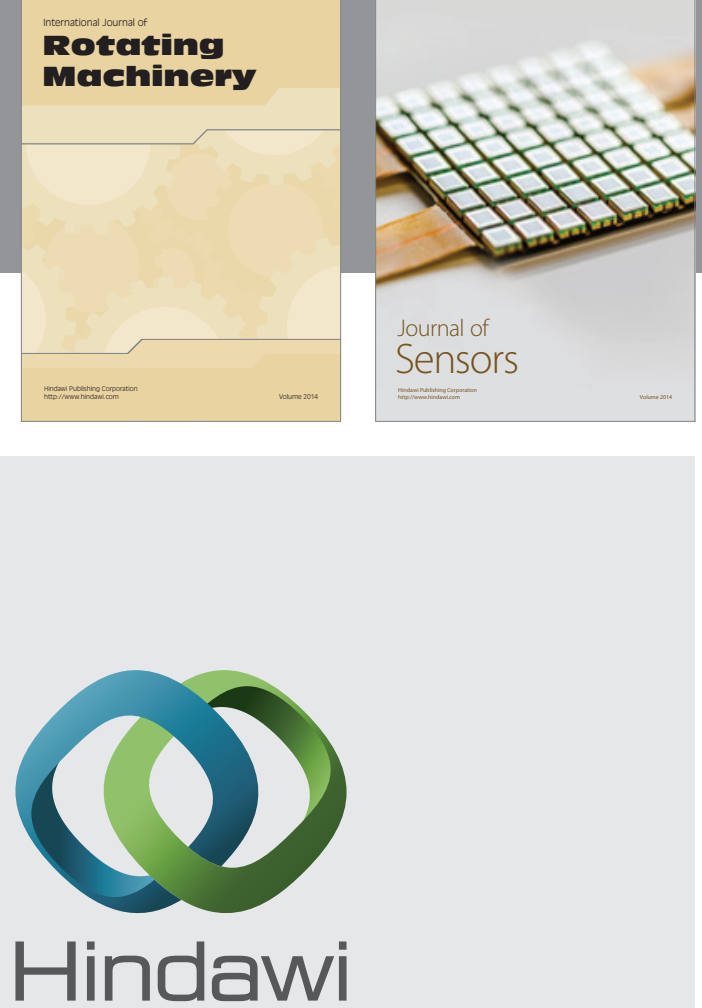

Submit your manuscripts at http://www.hindawi.com
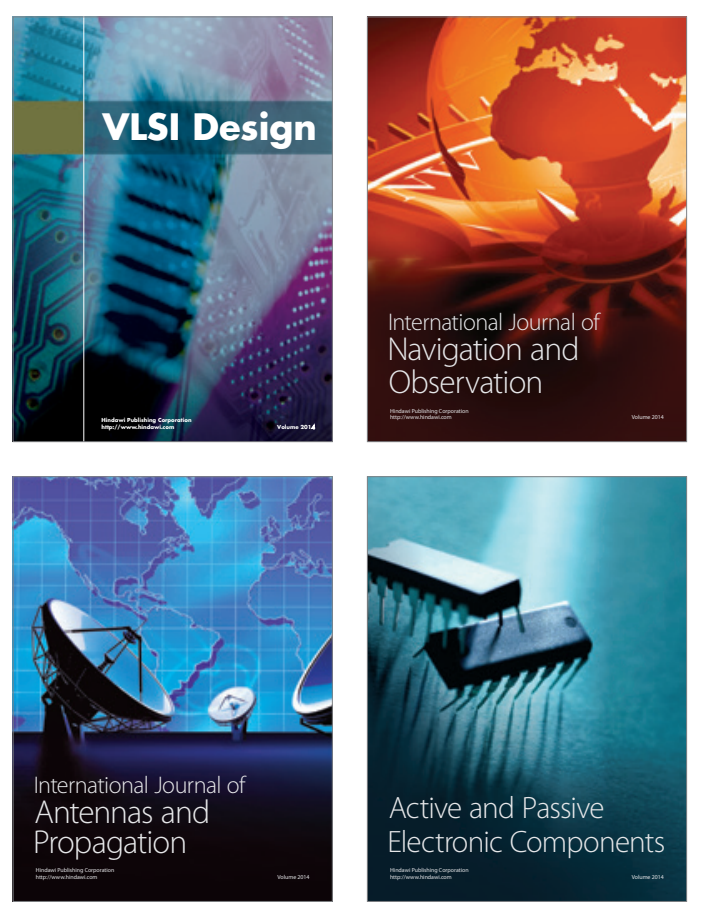
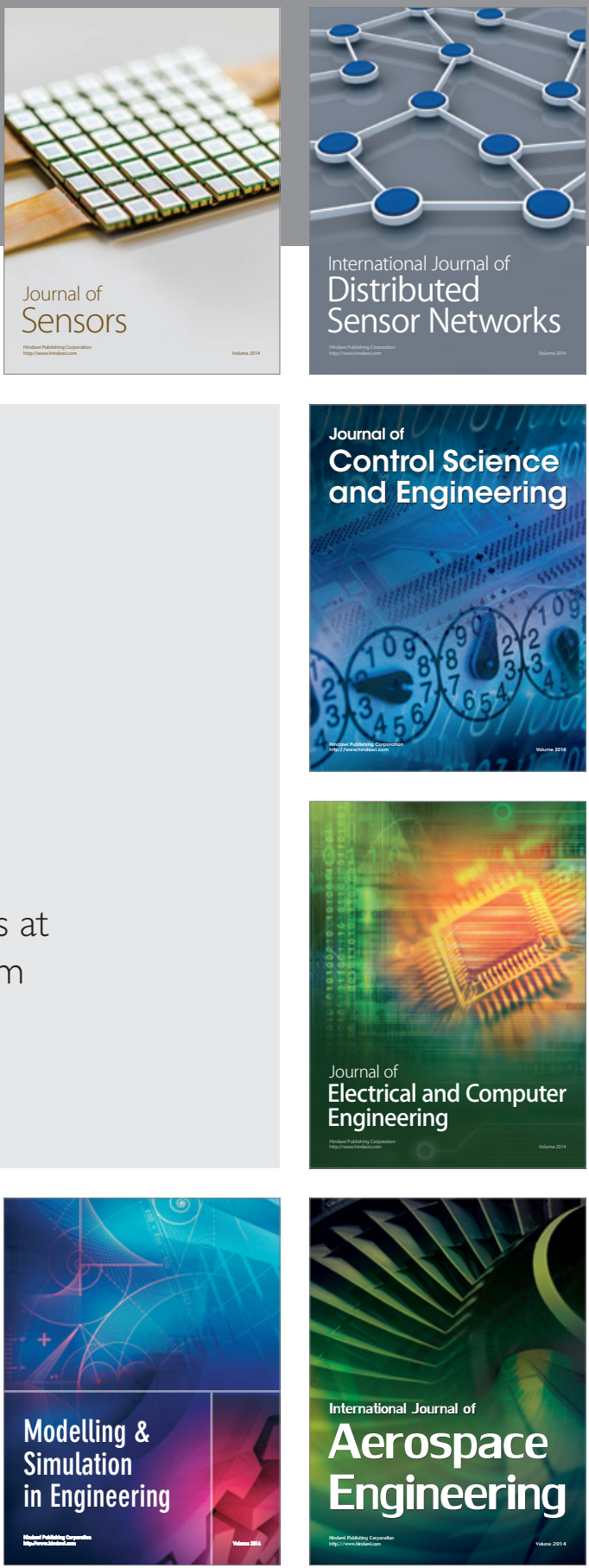

Journal of

Control Science

and Engineering
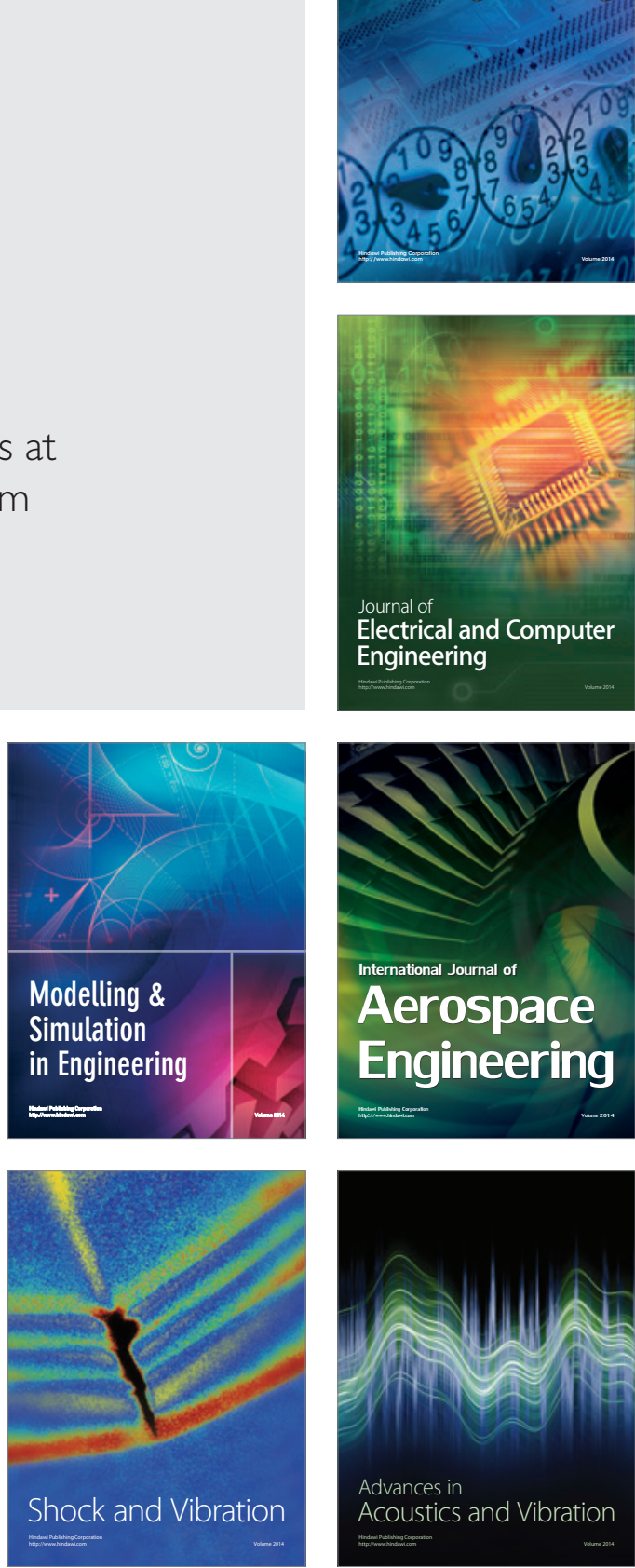